\title{
On the efficient treatment of temperature profiles for the estimation of atmospheric transmittance under scattering conditions
}

\author{
R. Lindstrot and R. Preusker \\ Institut für Weltraumwissenschaften, Freie Universität Berlin, Carl-Heinrich-Becker-Weg 6-10, 12165 Berlin, Germany \\ Correspondence to: R. Lindstrot (rasmus.lindstrot@wew.fu-berlin.de)
}

Received: 20 April 2012 - Published in Atmos. Meas. Tech. Discuss.: 29 June 2012

Revised: 21 September 2012 - Accepted: 24 September 2012 - Published: 26 October 2012

\begin{abstract}
The vertical temperature profile of the atmosphere has an influence on the width and intensity of gaseous absorption lines. In the visible and near infrared part of the spectrum, this poses a problem for the fast forward simulation of the radiative transfer, needed in algorithms for the retrieval of any atmospheric or surface-related parameter from satellite measurements. We show that the main part of the global variability of temperature profiles can be described by their first 2 to 6 eigenvectors, depending on the accuracy requirement, by performing a Principal Component Analysis (PCA) on a global set of temperature profiles from the Global Forecast System (GFS). Furthermore, we demonstrate the possibility to approximate the atmospheric transmittance in the $\mathrm{O}_{2} \mathrm{~A}$ band for any temperature profile with almost perfect accuracy by a linear combination of the transmittances attributed to each of the significant temperature eigenvectors. For the retrieval of surface pressure from $\mathrm{O}_{2} \mathrm{~A}$ band measurements, this reduces the global root mean square error from $>30 \mathrm{hPa}$ to better than $1 \mathrm{hPa}$ by strongly reducing the regional bias of surface pressure, retrieved on the assumption of an average temperature profile. The technique can be applied under scattering conditions to eliminate temperatureinduced errors in, e.g., simulated radiances. In principal, the method can be useful for any problem including gaseous absorption or emission with a significant influence of the temperature profile, such as the retrieval of total water vapour content or sea surface temperature.
\end{abstract}

\section{Introduction}

The forward simulation of the radiative transfer in the atmosphere is an essential component of any remote sensing algorithm for the retrieval of atmospheric or surface-related parameters from satellite observations. The requirements regarding the speed of the forward simulation can be hard to meet with state-of-the-art computers and radiative transfer models. This applies even more if both gaseous absorption and scattering from molecules, aerosols and/or clouds in the atmosphere are important, such as in gaseous absorption bands in the visible and near infrared part of the electromagnetic spectrum. Consequently, many forward simulation modules employed in retrieval algorithms in the shortwave region are based on pre-calculated look-up tables of the desired radiative quantity, which is tabulated as a function of the measurement geometry and any influencing geophysical parameter (see e.g. Gao and Kaufman, 2003; Schneising et al., 2009; Lindstrot et al., 2012).

Within absorption bands, the measurement is influenced by the vertical temperature profile through the impact of the pressure and temperature profile on the absorption line widths and intensities (Liou, 2002, see). The Doppler frequency shift caused by the molecular velocities results in a temperature-dependent line width, described by the Doppler line shape:

$f_{\mathrm{D}}\left(v-v_{0}\right)=\frac{1}{\alpha_{\mathrm{D}} \sqrt{\pi}} \exp \left[\frac{\left(v-v_{0}\right)^{2}}{\alpha_{\mathrm{D}}^{2}}\right]$

with the Doppler line width $\alpha_{\mathrm{D}} \propto T^{1 / 2}$. 
Additionally, absorption lines are broadened by molecular collisions, described by the Lorentz line shape:

$f_{\mathrm{L}}\left(v-v_{0}\right)=\frac{\alpha_{\mathrm{L}} / \pi}{\left(v-v_{0}\right)^{2}+\alpha_{\mathrm{L}}^{2}}$

with the Lorentz line width $\alpha_{\mathrm{L}}$, which is roughly proportional to the number of collisions per unit time: $\alpha_{\mathrm{L}} \propto p T^{-1 / 2}$.

The resulting line can approximately be described by the Voigt line shape, which is obtained from a convolution of both line profiles. In the lower atmosphere, the dominant process is pressure broadening with the resulting inverse influence of the temperature on the line width. An absorption line will therefore be broadened with increasing pressure and decreasing temperature, if the whole vertical column of the atmosphere is traversed.

It is not feasible to tabulate the simulated quantity as a function of the temperature in each vertical atmospheric layer, since this would result in look-up tables with an excessive, non-manageable number of dimensions. Such effort is indeed not necessary, since the individual layer temperatures are highly correlated and can be described with a much lower number of parameters, such as determined by means of a Principal Component Analysis (PCA, see e.g. Peixoto and Oort, 1992).

A PCA is an orthogonal linear transformation to a new coordinate system, such that the new variables are uncorrelated and the new coordinates carry the total variance in descending order. The technique is frequently used in various fields including atmospheric modeling and remote sensing. In the past, it has for example been applied with great success to reduce the dimensionality of hyperspectral simulations. Natraj et al. $(2005,2010)$ used PCA to eliminate redundancies in line-by-line simulations of, e.g. the $\mathrm{O}_{2} \mathrm{~A}$ band, by optimally grouping wavenumbers with similar optical properties. Liu et al. (2006) and Matricardi (2010) followed a related approach to speed up radiative transfer calculations for hyperspectral measurements from infrared sounders, such as the Infrared Atmospheric Sounding Interferometer (IASI) and the Atmospheric Infrared Sounder (AIRS). We propose using PCA to reduce the dimensionality of temperature profiles and reproduce the top-of-atmosphere (TOA) transmittance, exploiting the largely linear relationship between temperature profile eigenvectors and the transmittance.

The reconstruction of globally occurring temperature profiles is possible on the basis of a few eigenvectors, as demonstrated in Sect. 2.1. Furthermore, the atmospheric transmittance for an arbitrary temperature profile can be approximated in a similar way, as proposed in Sect. 2.2. This is demonstrated by applying the method to the retrieval of surface pressure from coarse spectral resolution $\mathrm{O}_{2}$ A band measurements and approximating high spectral resolution transmittance for a number of well-mixed and non well-mixed gases (see Sect. 3.1). The applicability for the approximation of radiances in the $\mathrm{O}_{2} \mathrm{~A}$ band is demonstrated in Sect. 3.2.
Table 1. Eigenvalues and (cumulative) percentage of variance for first 10 eigenvectors.

\begin{tabular}{cccc}
\hline Eigenvector & Eigenvalue & $\begin{array}{c}\text { Percent. } \\
\text { variance }\end{array}$ & $\begin{array}{c}\text { Cumul. percent. } \\
\text { of variance }\end{array}$ \\
\hline $\boldsymbol{v}_{1}$ & 3471 & $82.4 \%$ & $82.4 \%$ \\
$\boldsymbol{v}_{2}$ & 421 & $10.0 \%$ & $92.4 \%$ \\
$\boldsymbol{v}_{3}$ & 162 & $3.8 \%$ & $96.2 \%$ \\
$\boldsymbol{v}_{4}$ & 65 & $1.6 \%$ & $97.8 \%$ \\
$\boldsymbol{v}_{5}$ & 42 & $1.0 \%$ & $98.8 \%$ \\
$\boldsymbol{v}_{6}$ & 14 & $0.3 \%$ & $99.1 \%$ \\
$\boldsymbol{v}_{7}$ & 10 & $0.3 \%$ & $99.4 \%$ \\
$\boldsymbol{v}_{8}$ & 7 & $0.1 \%$ & $99.5 \%$ \\
$\boldsymbol{v}_{9}$ & 4 & $0.1 \%$ & $99.6 \%$ \\
$\boldsymbol{v}_{10}$ & 3 & $0.1 \%$ & $99.7 \%$ \\
$\ldots$ & $\ldots$ & $\ldots$ & $\ldots$ \\
\hline
\end{tabular}

\section{Statistical analysis}

\subsection{PCA of global temperature profiles}

The natural variability of the temperature profile was analysed using data from the Global Forecast System (GFS), providing the temperature on 26 pressure levels at a $1 \times 1$ degree resolution. A single, global field of temperature profiles from 19 October 2007 was used for the analysis. All following studies and plots are based on this case. Depending on the application, it might be necessary to use a more extended data set, such as one representing both the diurnal as well as the seasonal change of temperature profiles. Furthermore, it is beneficial to use data on an equal-area projection to avoid an overweighting of high latitudes. In the frame of this work, using a single day of GFS data is sufficient, since it merely serves to demonstrate the general validity of the approach.

Due to the high correlation of the temperature among neighbouring vertical layers, the number of independent parameters that suffice to reconstruct each vertical profile is significantly smaller than the number of pressure levels. A PCA was performed on the above mentioned global field of temperature profiles, revealing that the three most significant eigenvectors $\boldsymbol{v}_{j}$ are sufficient to reproduce $96 \%$ of the variability inherent to the data (see Table 1).

In case the statistical basis for the PCA is well-chosen, a random, realistic temperature profile $\boldsymbol{T}$ can be expressed as the sum of the average profile $\boldsymbol{T}_{\text {mean }}$ and a linear combination of the significant eigenvectors $\boldsymbol{v}_{j}$ :

$$
\boldsymbol{T}=\boldsymbol{T}_{\text {mean }}+\sum_{j=1}^{n \text { comp }} c_{j} \boldsymbol{v}_{j}
$$

where $n$ comp is the number of eigenvectors used. The weight $c_{j}$ of each eigenvector is obtained from $c_{j}=\left(\boldsymbol{T}-\boldsymbol{T}_{\text {mean }}\right)^{\top}$. $\boldsymbol{v}_{j}$.

Figure 1 shows three arbitrary profiles as extracted from GFS data and their reconstruction, using the three most 

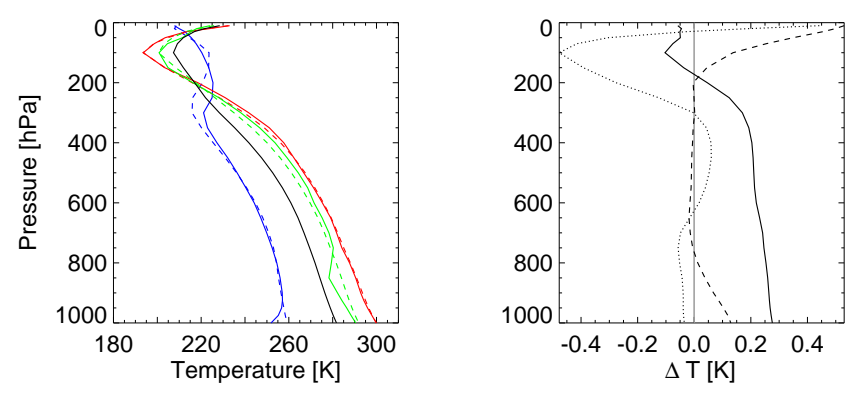

Fig. 1. Left panel: the mean profile ( $\boldsymbol{T}_{\text {mean }}$, black), three arbitrary temperature profiles (solid lines) and their reconstruction (dotted lines) using the three most significant eigenvectors. Right panel: first (solid), second (dotted) and third (dashed) eigenvector.

significant eigenvectors. While the dashed red curve shows an almost perfect representation of the vertical temperature profile, the green case reveals that more eigenvectors are needed to reconstruct the vertical fine structure, e.g. in cases of boundary layer temperature inversions. The blue case, representing an arctic atmosphere, shows that an extremely low tropopause height results in some deviations of the reconstructed profile. Here, more eigenvectors are needed to be able to reproduce the vertical temperature profile.

\subsection{Linearity between temperature eigenvectors and transmittance}

The strategy pursued in this work is based on the assumption that a linear relation between the temperature profile eigenvectors and the atmospheric transmittance exists. This means that the transmittance associated with any temperature profile can be constructed by a linear combination of the transmittance perturbations associated with the temperature profile eigenvectors, using the same weights $c_{j}$ as in Eq. (1). We define the effect of eigenvector $\boldsymbol{v}_{j}$ on the atmospheric transmittance, i.e. the first derivative $\frac{\delta t}{\delta \boldsymbol{v}_{j}}$, as:

$\Delta t_{\boldsymbol{v}_{j}}=\frac{\delta t}{\delta \boldsymbol{v}_{j}}=t_{\text {mean }+\boldsymbol{v}_{j}}-t_{\text {mean }}$

where $t_{\text {mean }}$ is the transmittance associated to the average temperature profile $\boldsymbol{T}_{\text {mean }}, t_{\text {mean }+\boldsymbol{v}_{j}}$ is the transmittance associated to the average profile, modified by eigenvector $\boldsymbol{v}_{j}$, and $\Delta t_{\boldsymbol{v}_{j}}$ is the resulting difference in transmittance for eigenvector $\boldsymbol{v}_{j}$, all for a given airmass. In accordance with Eqs. (1) and (2), the transmittance $t$ for a random temperature profile $\boldsymbol{T}$ is then constructed by a linear combination of the mean transmittance $t_{\text {mean }}$ and the contributions of each eigenvector $\Delta t_{v_{i}}$, using the same weights $c_{j}$ as determined for the temperature profile eigenvectors:

$t=t_{\text {mean }}+\sum_{j=1}^{n \text { comp }} c_{j} \Delta t_{\boldsymbol{v}_{j}}$.
Provided that the requirement of a linear relation between temperature eigenvectors and the transmittance is fulfilled, it is sufficient to calculate, for a given surface pressure, the transmittance $t_{\text {mean }}$ and $n$ comp partial derivatives of the transmittance, with respect to the temperature eigenvectors, to be able to construct the transmittance for any realistic temperature profile. If the transmittance-temperature-relation is non-linear, including the second derivative is suited to minimize the residual errors. The second derivative $\frac{\delta^{2} t}{\delta v_{j}^{2}}$ of $t$ with respect to eigenvector $\boldsymbol{v}_{j}$ can be approximated from

$\Delta^{2} t_{\boldsymbol{v}_{j}}=\frac{\delta^{2} t}{\delta v_{j}^{2}}=t_{\text {mean }+\boldsymbol{v}_{j}}+t_{\text {mean }-\boldsymbol{v}_{j}}-2 t_{\text {mean }}$.

Accordingly, the Taylor series (Eq. 3) is extended by the second derivative, neglecting the mixed partial derivatives:

$t=t_{\text {mean }}+\sum_{j=1}^{n \text { comp }} c_{j} \Delta t_{\boldsymbol{v}_{j}}+\sum_{j=1}^{n \text { comp }} c_{j}{ }^{2} \frac{\Delta^{2} t_{\boldsymbol{v}_{j}}}{2 !}$.

A detailed mathematical description is provided in the Appendix.

The technique has the potential to speed up the calculation of atmospheric transmittance and enable the inclusion of the temperature profile effect without the need to perform the computationally demanding calculation of absorption coefficients for each temperature profile under consideration. The validity of the approach is demonstrated in Sect. 3 for the retrieval of surface pressure from coarse spectral resolution measurements in the $\mathrm{O}_{2} \mathrm{~A}$ band. Furthermore, the applicability is tested at high spectral resolution for a variety of wellmixed and non well-mixed gases.

\section{Application}

\subsection{Transmittance approximation}

\subsubsection{Coarse spectral resolution}

In order to test the approach for the approximation of atmospheric transmittance, the global set of GFS temperature profiles was used to calculate the average $\mathrm{O}_{2} \mathrm{~A}$ band transmittance $t_{\mathrm{O}_{2 \mathrm{~A}}}$ between $760 \mathrm{~nm}$ and $763 \mathrm{~nm}$ for a fixed solar zenith angle of $45^{\circ}$ and nadir view, hereby using the HITRAN 2008 database (Rothman et al., 2009). The problem was simplified by assuming a globally constant surface pressure of $1000 \mathrm{hPa}$, neglecting surface elevation in order to isolate the effect of temperature broadening on the $\mathrm{O}_{2}$ absorption lines (see Fig. 2 for an example of the transmittance in the considered spectral window). The resulting global transmittance for the considered case is shown in in Fig. 3. The highest transmittance, respectively weakest $\mathrm{O}_{2}$ absorption, is found in regions with the highest temperatures, namely over tropical land areas. As stated before, this is due 


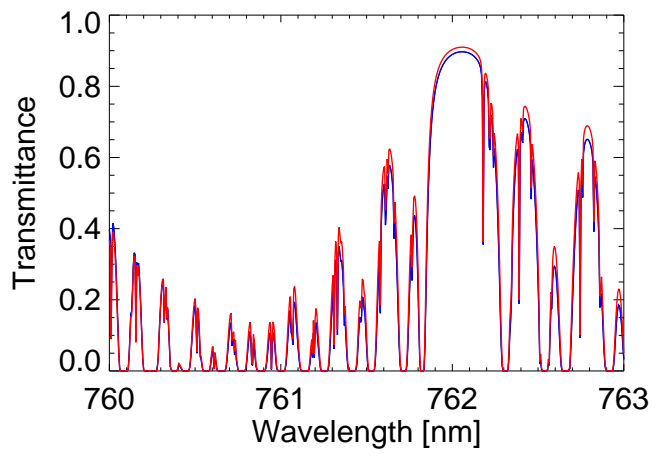

Fig. 2. Atmospheric transmittance in $\mathrm{O}_{2} \mathrm{~A}$ band for an arctic (blue) and a tropical temperature profile (red), a surface pressure of $1000 \mathrm{hPa}$, a solar zenith of $45^{\circ}$ and nadir view.

to the pressure- and temperature-dependence of the Lorentz line widths, resulting in less broadened absorption lines for higher temperatures. The hypothetical transmittance for the given airmass of 2.41 varies between 0.227 in the Antarctic and 0.265 in the tropics.

For comparison, a transmittance $t_{\mathrm{O}_{2 \mathrm{~A}}}^{*}$ was obtained by a linear combination of the eigenvector contributions, following formula (3). The global root mean square deviation of the derived transmittance is shown in Fig. 4 as a function of the number of eigenvectors used for the approximation of transmittance. The error is reduced from roughly $1 \%$ of transmittance, in case an average temperature profile is assumed, to $0.09 \%$, by just using the first temperature profile eigenvector to modify the average transmittance. Adding the second eigenvector yields a further reduction of the transmittance error below $0.05 \%$. The error can be brought down to $0.015 \%$ by using 6 eigenvectors, with this residual error indicating the limit of the approach caused by the simplifying assumption of linearity.

Figure 5 shows the global distribution of the errors of $t_{\mathrm{O}_{2 \mathrm{~A}}}^{*}$ as a function of the number of eigenvectors used. In the case of using only the most significant eigenvector, some systematic deviations of the approximated transmittance are apparent, with positive biases found mainly over Antarticta and Africa and negative biases found mainly over the extratropical oceans. These systematic biases are gradually eliminated with increasing numbers of eigenvectors and are almost totally removed in the case four or more eigenvectors are used.

As an example, satellite measurements in the $\mathrm{O}_{2} \mathrm{~A}$ band can be used for the retrieval of surface pressure, as demonstrated by e.g. Barton and Scott (1986), Mitchell and O'Brien (1987), Breon and Bouffies (1996) and O'Brien et al. (1998). Lindstrot et al. (2009) have developed a corresponding algorithm based on such measurements performed by the Medium Resolution Imaging Spectrometer (MERIS) onboard ENVISAT. However, the initial algorithm version is based on the assumption of a fixed, average temperature

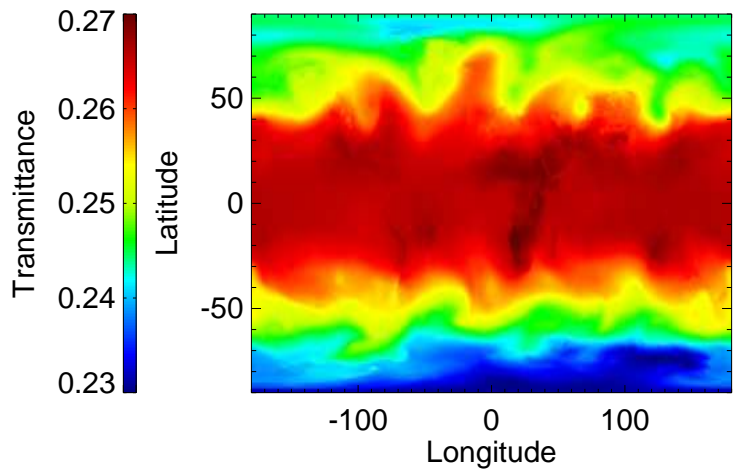

Fig. 3. Global transmittance $t_{\mathrm{O}_{2 A}}$ for GFS temperature profiles, a constant surface pressure of $1000 \mathrm{hPa}$, solar zenith angle of $45^{\circ}$ and nadir view.

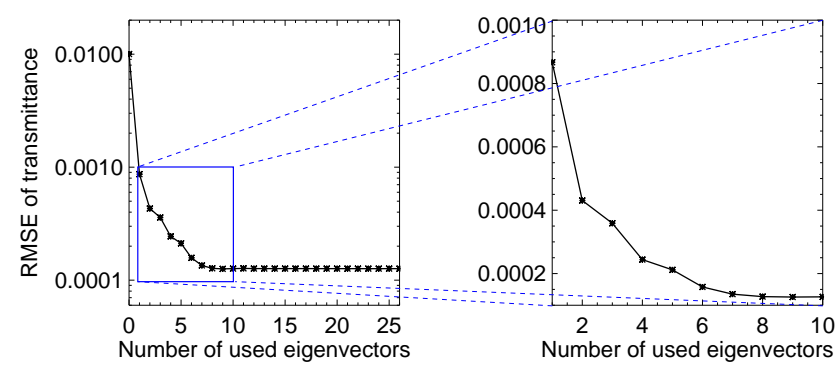

Fig. 4. Global root mean square error of approximated transmittance between 760 and $763 \mathrm{~nm}$ for a surface pressure of $1000 \mathrm{hPa}$ and an airmass of 2.41, as a function of the number of eigenvectors used.

profile, resulting in regional surface pressure biases of up to $50 \mathrm{hPa}$, as shown in Fig. 6, left panel.

If the temperature profile variability is accounted for using the technique described above, these large systematic errors can be eliminated, as shown in Fig. 6, right panel. The corresponding global root mean square error of surface pressure due to temperature profile effects is reduced from $35 \mathrm{hPa}$ to less than $0.5 \mathrm{hPa}$, if six or more eigenvectors are used (see Fig. 7). Other error sources, such as the uncertainty due to aerosol loading ( $\sim 10 \mathrm{hPa}$ if a mean optical depth is assumed) or the MERIS spectral calibration ( $0-50 \mathrm{hPa}$ for a calibration uncertainty of $0.1 \mathrm{~nm}$, depending on channel position), result in considerable errors in derived surface pressure (Lindstrot et al., 2009, 2010). Therefore, using two eigenvectors to describe the temperature profile variability is sufficient to account for the temperature-dependence of the $\mathrm{O}_{2}$ absorption for the case of surface pressure retrievals from coarse spectral resolution measurements in the $\mathrm{O}_{2}$ A band, since the accepted error of $\sim 1.5 \mathrm{hPa}$ is well below the errors introduced by other sources of uncertainty. 

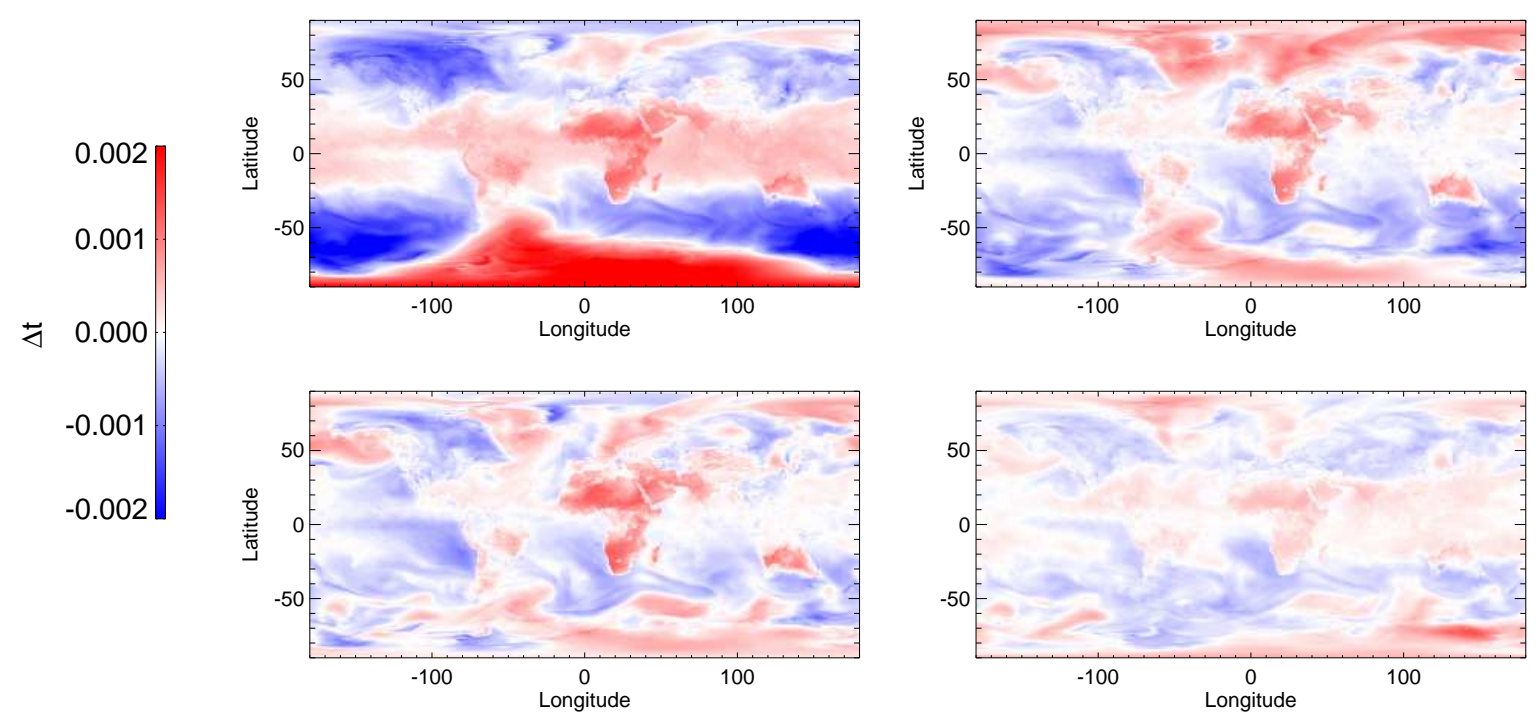

Fig. 5. Global distribution of bias of approximated transmittance $t_{\mathrm{O}_{2 \mathrm{~A}}}^{*}$, using 1, 2, 3 or 4 eigenvectors (upper left to lower right).
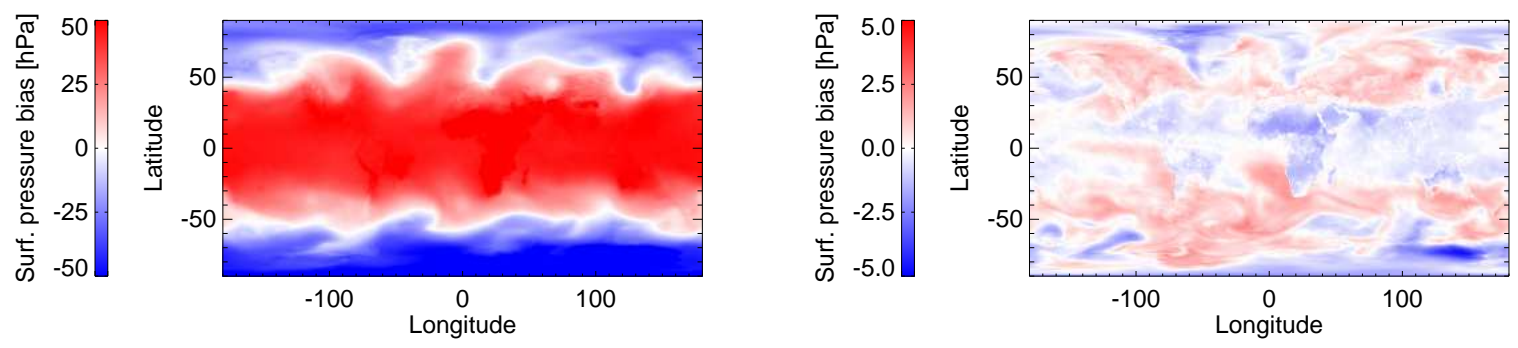

Fig. 6. Global distribution of bias of retrieved surface pressure for a fixed average temperature profile (left) and in the case four temperature eigenvectors are used for the approximation of the transmittance (right), shown for a constant surface pressure of $1000 \mathrm{hPa}$ and an airmass of 2.41. Note the different color bars.
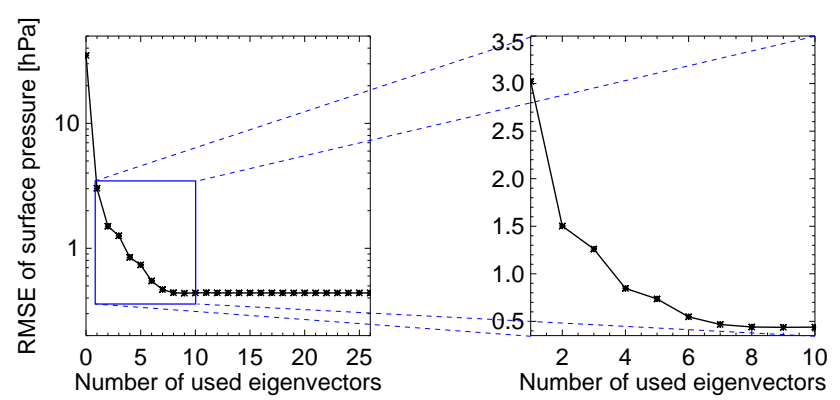

Fig. 7. Global root mean square error of surface pressure, derived from approximated transmittance, as a function of the number of eigenvectors used.

\subsubsection{High spectral resolution}

In the previous section, the technique was tested at coarse spectral resolution for the well-mixed gas $\mathrm{O}_{2}$. In order to evaluate the validity of the approach for different at- mospheric species and at high spectral resolution, a close look was taken at the hyperspectral transmittance in a variety of spectral intervals. Figure 8 shows the transmittance and the associated error in the approximated transmittance for a random tropical profile for absorption lines of $\mathrm{O}_{2}(762 \mathrm{~nm}-764 \mathrm{~nm}), \mathrm{CO}_{2}(2008 \mathrm{~nm}-2010 \mathrm{~nm}), \mathrm{CH}_{4}$ $(3201 \mathrm{~nm}-3208 \mathrm{~nm})$ and water vapour $(900 \mathrm{~nm}-902 \mathrm{~nm}$, $940 \mathrm{~nm}-942 \mathrm{~nm}, 1780 \mathrm{~nm}-1782 \mathrm{~nm}$ ). Both the linear correction (see Eq. 3) as well as the correction including the second derivative (see Eq. 5) are shown. The main conclusions are as follows:

- For $\mathrm{O}_{2}$ absorption (panel $A$ ), the initial error in transmittance, caused by assuming an average temperature profile, reaches maxima at the line flanks in the range of 0.03-0.04 of absolute transmittance. Applying the linear correction (using 4 eigenvectors) results in small residual errors below 0.005 . Accounting for the second derivative does not improve the accuracy of the approximated transmittance. 
- Similar numbers are found for $\mathrm{CO}_{2}(\operatorname{panel} B)$. However, here the second-derivative correction improves the accuracy, with the residual error in transmittance being reduced by half. This hints at a weak non-linearity between $\boldsymbol{v}_{j}$ and $t$.

- The situation for $\mathrm{CH}_{4}$ (panel $C$ ) is similar to the $\mathrm{CO}_{2}$ case.

- For water vapour, a non well-mixed gas, generally the errors in transmittance are larger in comparison to the other gases. This is in part due to the fact that the maximum temperature differences between $\boldsymbol{T}_{\text {mean }}$ and the temperature profile under consideration are found in lower atmosphere, as is the bulk of water vapour. In the $\rho \sigma \tau$-absorption band, the intial errors are up to 0.1 with peaks around the centers of unsaturated absorption lines. Using the principal components eliminates the errors, except for some spectral ranges, such as found at $901 \mathrm{~nm}$ (panel $D$ ) or $940.6 \mathrm{~nm}$ (panel $E$ ). Here, including the second derivative further improves the accuracy of the approximated transmittance.

- The largest errors are found for water vapour absorption around $1780 \mathrm{~nm}$ (panel $F$ ): in the line wings, the transmittance is overestimated by up to 0.2 , when the average profile is assumed, with the peaks of the errors located in the centers of unsaturated and the edges of saturated absorption lines. Correcting for the linear effect of four eigenvectors is sufficient to eliminate large parts of the deviation, however, those regions with the highest initial errors are still not corrected completely (see $1781.6 \mathrm{~nm}-$ $1781.9 \mathrm{~nm}$ range). Including the second derivative for correction further improves the approximation.

The residual errors of the approximated transmittance, mainly found in regions of large optical depth, raise the question whether better results can be obtained by applying the PCA in optical depth-space rather than reproducing the transmittance directly. This means that instead of the scalar transmittance $t$, the profile of the optical depth $\tau$ is approximated and the transmittance is calculated afterwards. Consequently, Eqs. (2)-(5) become:

$$
\begin{array}{r}
\Delta \boldsymbol{\tau}_{\boldsymbol{v}_{j}}=\frac{\delta \boldsymbol{\tau}}{\delta \boldsymbol{v}_{j}}=\boldsymbol{\tau}_{\text {mean }+\boldsymbol{v}_{j}}-\boldsymbol{\tau}_{\text {mean }} \\
\boldsymbol{\tau}=\boldsymbol{\tau}_{\text {mean }}+\sum_{j=1}^{n c o m p} c_{j} \Delta \boldsymbol{\tau}_{\boldsymbol{v}_{j}}
\end{array}
$$

and, if the second derivative is included

$$
\begin{array}{r}
\Delta^{2} \boldsymbol{\tau}_{\boldsymbol{v}_{j}}=\frac{\delta^{2} \boldsymbol{\tau}}{\delta \boldsymbol{v}_{j}^{2}}=\boldsymbol{\tau}_{\text {mean }+\boldsymbol{v}_{j}}+\boldsymbol{\tau}_{\text {mean }-\boldsymbol{v}_{j}}-2 \boldsymbol{\tau}_{\text {mean }} \\
\boldsymbol{\tau}=\boldsymbol{\tau}_{\text {mean }}+\sum_{j=1}^{n \text { comp }} c_{j} \Delta \boldsymbol{\tau}_{\boldsymbol{v}_{j}}+\sum_{j=1}^{n \text { comp }} c_{j}{ }^{2} \frac{\Delta^{2} \boldsymbol{\tau}_{\boldsymbol{v}_{j}}}{2 !}
\end{array}
$$

Then, the transmittance is calculated as usual, with

$t=\exp \left(-\sum_{i=1}^{n} \tau_{i} m\right)$

where $m$ denotes the airmass.

Comparing Figs. 8 and 9 allows for the following conclusions:

- For $\mathrm{O}_{2}, \mathrm{CO}_{2}$ and $\mathrm{CH}_{4}$, no considerable improvement is found when the gaseous optical depth profile is reproduced instead of the transmittance.

- The accuracy of the approximated transmittance is improved in regions of water vapour absorption, at least in the $1780 \mathrm{~nm}$ region. Here, the error of the approximated transmittance (including the second derivative) is improved from $-0.0034 \pm 0.0056$ to $-0.0011 \pm 0.001$. The residuals in regions of high optical depth are erased.

At least for water vapour absorption, it therefore seems advantageous to act in $\tau$-space. Generally, it is important to note that the influence of the temperature profile on the transmittance differs among the individual atmospheric constituents and the considered spectral ranges. It is therefore crucial to assess the needed number of eigenvectors and the optimal correction approach for each individual application. A further improvement of the results can be expected from including the mixed partial derivatives in Eqs. (5) and (9), respectively, rather than from increasing the number of eigenvectors used. Furthermore, the required accuracy of reproduced transmittance spectra is constrained by the uncertainty of the assumed temperature profile and the associated error in transmittance. For the water vapour case, temperature profiles extracted from NWP models are likely to exhibit some uncertainty in cases of boundary layer temperature inversions, causing an uncertainty in the derived absorption coefficients and resulting transmittance. Reliable estimates of this uncertainty can help to identify the required accuracy of the transmittance approximation and thus the adequate correction strategy.

\subsection{Radiance approximation}

We have demonstrated the validity of the approach for the approximation of transmittance in the $\mathrm{O}_{2} \mathrm{~A}$ band. We hypothesize that the technique can be as equally well used under scattering conditions to approximate the TOA radiances for any given temperature profile from the corresponding TOA radiances associated with each temperature profile eigenvector. By replacing the transmittance $t$ with radiance $L$, Eq. (3) becomes:

$$
L_{n \text { comp }}=L_{\text {mean }}+\sum_{j=1}^{n \text { comp }} c_{j} \Delta L_{\boldsymbol{v}_{j}}
$$



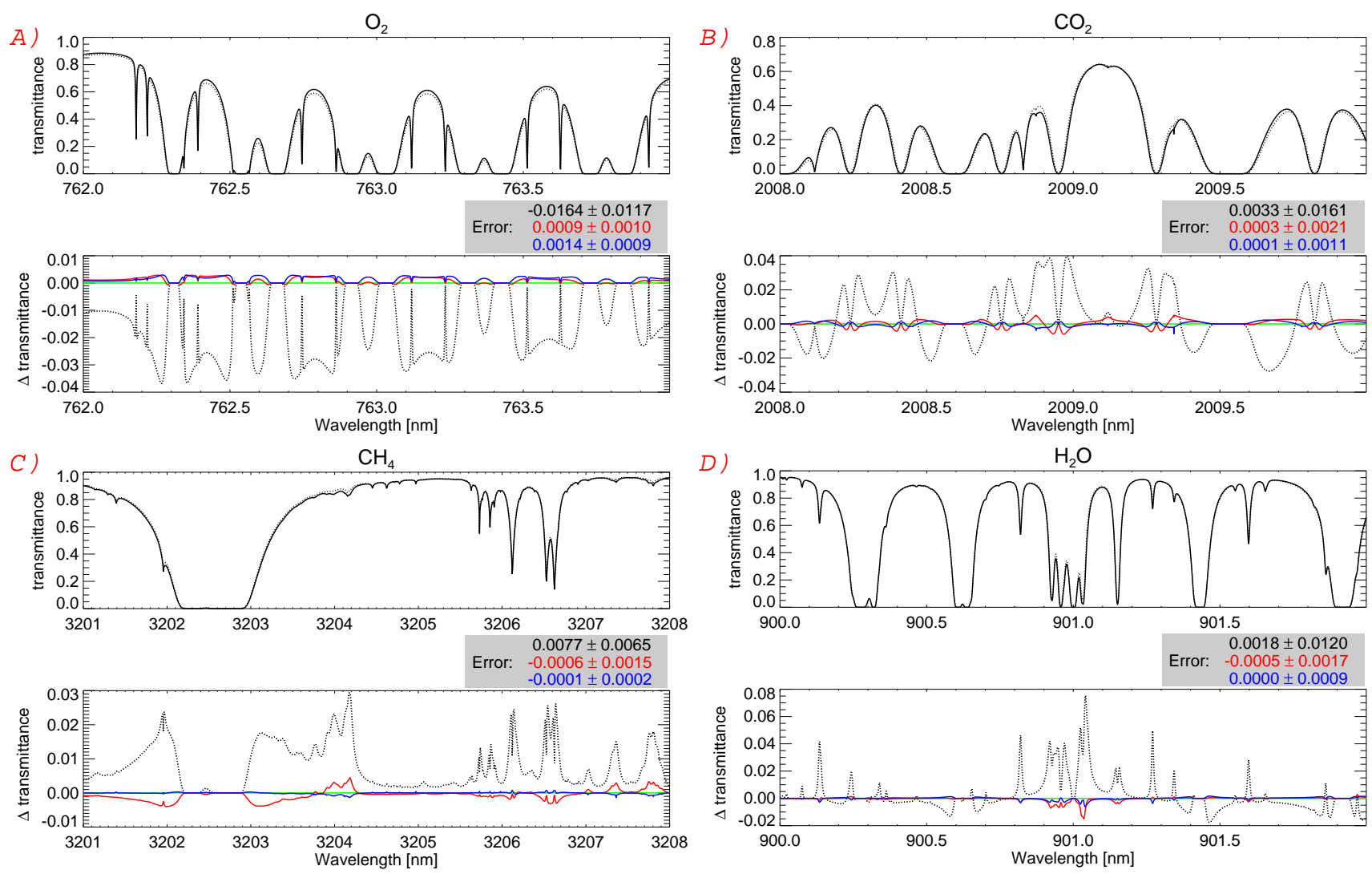

E) $\mathrm{H}_{2} \mathrm{O}$

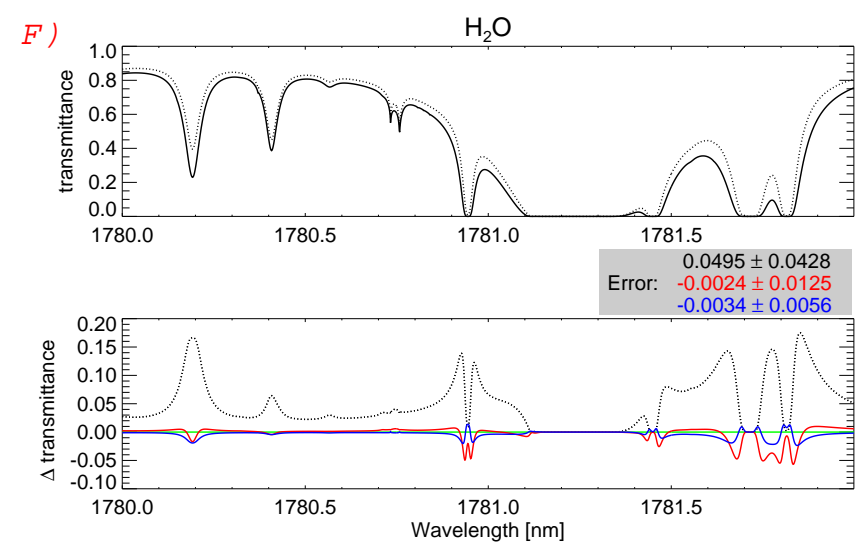

Fig. 8. Transmittance for tropical atmosphere (airmass =3, upper panels) and accuracy of approximated transmittance (lower panels) for various gases $\left(\mathbf{A}: \mathrm{O}_{2}, \mathbf{B}: \mathrm{CO}_{2}, \mathbf{C}: \mathrm{CH}_{4}, \mathbf{D}-\mathbf{F}\right.$ : water vapour). Black dotted lines show errors in the case average temperature profile is used, red and blue lines indicate errors when using 4 principal components, (red: first derivative only, blue: including second derivative). The associated accuracy and precision of the approximated transmittance are given in the grey shaded boxes, calculated from the spectral ranges shown.

where of course $\Delta L_{\boldsymbol{v}_{j}}$ denotes the sensitivity of the radiance $L$ to eigenvector $\boldsymbol{v}_{j}$ :

$\Delta L_{\boldsymbol{v}_{j}}=\frac{\delta L}{\delta \boldsymbol{v}_{j}}=L_{\text {mean }+\boldsymbol{v}_{j}}-L_{\text {mean }}$

This means that the radiance for any atmospheric profile, observing geometry and geophysical conditions, can be represented by a linear combination of the tabulated $L_{\text {mean }}$ and $n$ comp radiances.

In order to test this hypothesis, we have performed full radiative transfer calculations of a MERIS-like mediumspectral-resolution channel within the $\mathrm{O}_{2} \mathrm{~A}$ band, using the Matrix Operator Model (MOMO, Fell and Fischer, 2001; Hollstein and Fischer, 2012). The spectral channel was assumed to have a Gaussian shape with a width of $3 \mathrm{~nm}$ and 

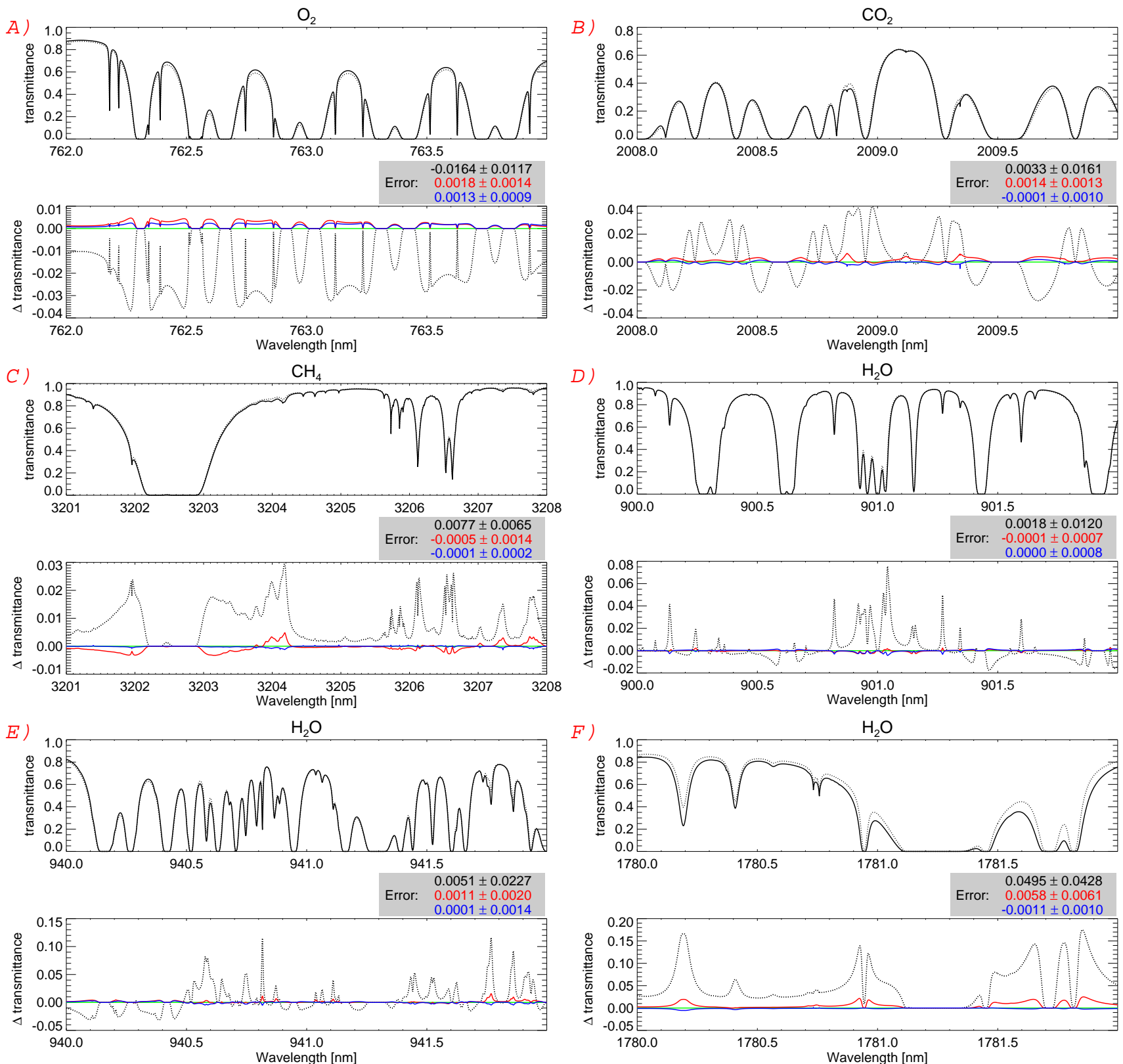

Fig. 9. Same as for Fig. 8, but applying the PCA in optical depth space and calculcating the transmittance afterwards.

shifted through the absorption band to visualize the spectral stability of the approach. In order to isolate the effect of scattering on the accuracy of the method, two general cases were studied:

A. A case with a strong influence of atmospheric scattering, i.e. a high aerosol loading (aerosol optical depth $\mathrm{AOD}_{550 \mathrm{~nm}}=0.5$ ) over dark Lambertian surface (albedo $\alpha=0.1$ ).

B. A case with a weaker influence, i.e. a low aerosol loading $\left(\mathrm{AOD}_{550 \mathrm{~nm}}=0.1\right)$, over a bright Lambertian surface $(\alpha=0.5)$.
The approximation of the TOA radiance was performed following Eq. (11) for a tropical and a polar temperature profile with a surface pressure of $1000 \mathrm{hPa}$ (see red and blue profiles in Fig. 1). Figure 10 shows the angular dependency of the differences found between the TOA radiances simulated using the average temperature profile and the tropical profile. If the average temperature profile is used, the deviation of the TOA radiance from the truth, $\Delta L_{\text {mean }}$, is between $-4 \%$ (Case A) and $-3 \%$ (Case B) for low zenith angles (sensor and sun close to zenith) and rises to $-8 \%$ (Case A) and $-7 \%$ (Case B) for high angles. Similar numbers with reversed sign 

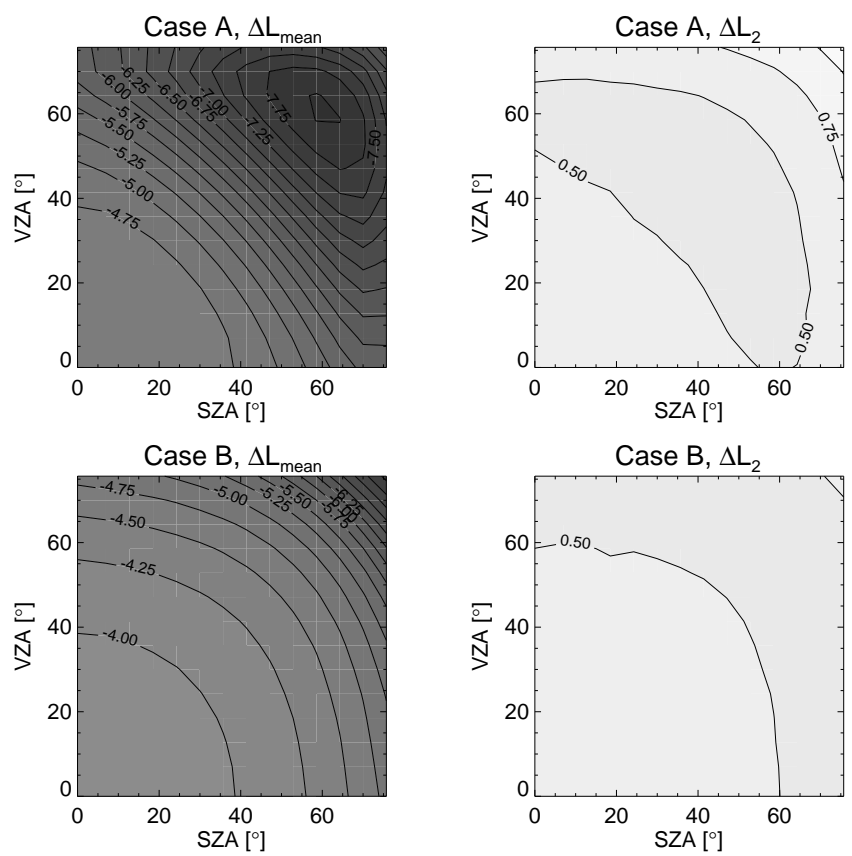

Fig. 10. Angular dependence of relative error in $\%$ of TOA radiance for the tropical profile for cases A (upper panels) and B (lower panels), a $3 \mathrm{~nm}$-wide channel located at $763 \mathrm{~nm}$ and the sensor placed opposite of the sun (relative azimuth angle $0^{\circ}$ ). Left column shows $\Delta L_{\text {mean }}$, i.e. the error in case the average profile is used; right column shows $\Delta L_{2}$, i.e. the error in case the first two eigenvectors are used. The contour lines have a distance of $0.25 \%$ in all plots.

are found for the polar case. When the first two eigenvectors are used for correcting the radiance, the error, $\Delta L_{2}$, is well below $1 \%$ for both cases at all viewing geometries. There is no significant influence of the observation geometry on the accuracy of the method, which is able to eliminate the overall bias and its angular dependence.

Figure 11 shows the influence of the number of eigenvectors on the approximation error. For both cases and both profiles, the initial error of several percent is reduced to well below $1 \%$ by using the first temperature eigenvector and slightly improved by accounting for the second eigenvector. The addition of further eigenvectors does not significantly change the result. The elimination of the temperatureinduced radiance bias works for both cases with the same accuracy, indicating that for this purpose atmospheric scattering is not a critical issue.

While $\Delta L_{\text {mean }}$ shows a strong dependency on the central wavelength with a clear maximum around $762.5 \mathrm{~nm}, \Delta L_{2}$ is close to zero and the systematic spectral signature is replaced by an apparently random residual (see Fig. 11). Since, depending on the instrument construction type, the central wavelengths of spectral channels can vary with time or the view angle (as an example see Delwart et al., 2007), it is important to account for these spectral effects to avoid temporal or view angle dependent biases in derived products.
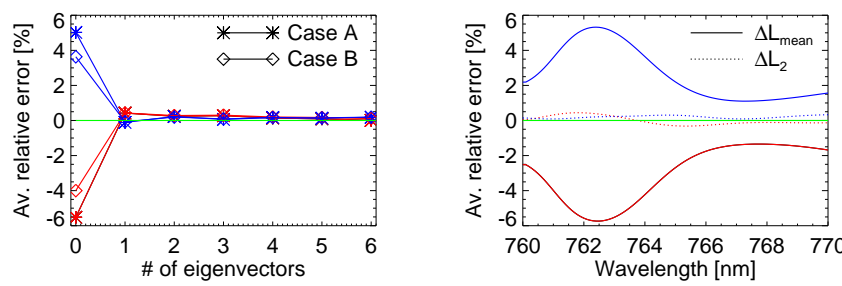

Fig. 11. Left panel: error of approximated radiance (average over all viewing geometries) as a function of number of eigenvectors used, for both cases and both profiles (red lines: tropical profile, blue lines: polar profile) and a spectral channel located at $763 \mathrm{~nm}$. Right panel: error of $L_{\text {mean }}$ (solid lines) and $L_{2}$ (dotted lines) for case A (average over all viewing geometries), as a function of wavelength.

\section{Conclusions}

We have presented a method to efficiently approximate the effect of the temperature profile on the atmospheric transmittance. The first two to six eigenvectors are sufficient to describe the global variability of temperature profiles, depending on what accuracy is required. The temperature eigenvectors can serve to calculate the corresponding variations in optical depth or transmittance directly and thus to approximate the atmospheric transmittance for any realistic temperature profile.

As shown in an exemplary application for the case of $\mathrm{O}_{2}$ absorption around $0.76 \mu \mathrm{m}$, the such approximated transmittance is in almost perfect agreement with the truth if a sufficient number of eigenvectors is used. In the shown case, an average $\mathrm{O}_{2}$ transmittance of 0.25 between $760 \mathrm{~nm}$ and $763 \mathrm{~nm}$ for an airmass of 2.41 and a surface pressure of $1000 \mathrm{hPa}$, the residual error of the approximated transmittance is in the range of 0.0015 in absolute transmittance, which is $0.06 \%$. At high spectral resolution, it could be demonstrated that the technique works to strongly reduce the bias occurring in transmittance calculated for the average temperature profile. For some gases, such as water vapour, including the second derivative of transmittance with respect to the eigenvectors can improve the approximation accuracy. Furthermore, for water vapour the optimal correction strategy was found to be a reproduction of the optical depth profile rather than the transmittance directly.

Depending on the nature of the application, the number of eigenvectors needed may vary. As demonstrated for the retrieval of surface pressure from MERIS, using two eigenvectors is sufficient since the resulting residual error of $\sim 1.5 \mathrm{hPa}$ is much smaller than that of other error sources. The technique can readily be used for the elimination of any temperature-induced bias in simulated radiances. Scattering at molecules and particles does not affect the applicability or accuracy of the method. 
The benefit of the presented method can either be seen as

- a major speed up of the calculation of transmittance or radiance, for which the method works with sufficient accuracy, in any forward simulation module used to model radiative transfer in atmospheric absorption bands, or

- the avoidance of temperature-induced errors in calculated radiances at the cost of a linear increase of the amount of simulations to be performed.

It is important to state that the number of eigenvectors needed and the linearity between temperature profile eigenvectors and the transmittance need to be assessed for each potential application, since the transmittance exhibits differing sensitivities to the temperature profile for different atmospheric species and spectral ranges. An upper boundary for the required accuracy of the reproduced transmittances is given by the uncertainty resulting from the limited knowledge of the temperature profile itself, that might be provided by NWP models or temperature soundings.

\section{Appendix A}

Let $F: \mathbb{R}^{n} \rightarrow \mathbb{R}$ be a function which is partially differentiable at $\boldsymbol{x}$. The best linear approximation of $F$ near $\boldsymbol{x}$ is:

$$
\begin{aligned}
F(\boldsymbol{x}+\Delta \boldsymbol{x}) & \approx F(\boldsymbol{x})+\frac{\delta F}{\delta x_{1}} \Delta x_{1}+\ldots+\frac{\delta F}{\delta x_{n}} \Delta x_{n} \\
& =F(\boldsymbol{x})+\sum_{i=1}^{n} \frac{\delta F}{\delta x_{i}} \Delta x_{i}
\end{aligned}
$$

if $F$ is totally differentiable at $\boldsymbol{x}$. Best linear approximation means that:

$$
\lim _{\Delta x \rightarrow 0}\left(\frac{F(x+\Delta x)-F(x)-\nabla F \cdot \Delta x}{\|\Delta x\|}\right)=0
$$

with

$\mathrm{d} F=\nabla F \cdot \Delta \boldsymbol{x}=\sum_{i=1}^{n} \frac{\delta F}{\delta x_{i}} \Delta x_{i}$

being the total derivative of $F$. Necessary and sufficient conditions for total differentiability are, that all partial derivatives exist and that they are continuous. In our case, where $F$ is the atmospheric transmission $t$ as a function of the air temperature at different levels $\boldsymbol{T}$, the conditions are fulfilled, since there are no discontinuities in $t$ due to small changes of $\boldsymbol{T}$. The total derivative of $t$ is:

$\mathrm{d} t=\sum_{i=1}^{n} \frac{\delta t}{\delta T_{i}} \Delta T_{i}$. is:

Consequently, the linear aproximation of $t$ at $\boldsymbol{T}_{\text {mean }}+\Delta \boldsymbol{T}$

$t\left(\boldsymbol{T}_{\text {mean }}+\Delta \boldsymbol{T}\right) \approx t\left(\boldsymbol{T}_{\text {mean }}\right)+\sum_{i=1}^{n} \frac{\delta t}{\delta T_{i}} \Delta T_{i}$.
In particular, when $\boldsymbol{T}_{\text {mean }}$ is changed by an eigenvector $\boldsymbol{v}_{j}$ (see Eq. 2), the approximation is:

$t\left(\boldsymbol{T}_{\text {mean }}+\boldsymbol{v}_{j}\right)-t\left(\boldsymbol{T}_{\text {mean }}\right)=\Delta t_{\boldsymbol{v}_{j}} \approx \sum_{i=1}^{n} \frac{\delta t}{\delta T_{i}} v_{i, j}$.

A principal component analysis is an orthogonal linear transformation to a new coordinate system, such that the new variables are uncorrelated, and that the new coordinates carry the total variance in descending order. A PCA can be done by an eigenvector decomposition of the variance-covariance matrix of the mean-centered data, whereat the eigenvectors define the new coordinate system. In this work, the atmospheric temperature profiles $\boldsymbol{T}$ are decomposed:

$\boldsymbol{T}-\boldsymbol{T}_{\text {mean }}=\sum_{j=1}^{n} c_{j} \boldsymbol{v}_{j}$

with the eigenvectors $\boldsymbol{v}_{j}$ and the projections in the eigenspace

$c_{j}=\left(\boldsymbol{T}-\boldsymbol{T}_{\text {mean }}\right)^{\top} \cdot \boldsymbol{v}_{j}$.

If the original coordinate system (here the temperature at different levels) has correlated variables, the PCA can be used for a dimensionality reduction ( $n \rightarrow n$ comp) without losing significant information.

$\boldsymbol{T}-\boldsymbol{T}_{\text {mean }} \approx \sum_{j=1}^{n \text { comp }} c_{j} \boldsymbol{v}_{j}$

Combining Eqs. (A5) and (A9) yields:

$$
\begin{aligned}
t\left(\boldsymbol{T}_{\text {mean }}+\Delta \boldsymbol{T}\right)-t\left(\boldsymbol{T}_{\text {mean }}\right) & \approx \sum_{i=1}^{n} \frac{\delta t}{\delta T_{i}} \sum_{j=1}^{n \text { comp }} c_{j} v_{i, j} \\
& =\sum_{i=1}^{n} \sum_{j=1}^{n \text { comp }} \frac{\delta t}{\delta T_{i}} c_{j} v_{i, j} \\
& =\sum_{j=1}^{n \text { comp }} \sum_{i=1}^{n} \frac{\delta t}{\delta T_{i}} c_{j} v_{i, j} \\
& =\sum_{j=1}^{n \text { comp }} c_{j} \sum_{i=1}^{n} \frac{\delta t}{\delta T_{i}} v_{i, j}
\end{aligned}
$$

Replacing the right sum using Eq. (A6) results in:

$$
t\left(\boldsymbol{T}_{\text {mean }}+\Delta \boldsymbol{T}\right)-t\left(\boldsymbol{T}_{\text {mean }}\right) \approx \sum_{j=1}^{n \text { comp }} c_{j} \Delta t_{v_{j}},
$$

which is equivalent to Eq. (3). Eventually, it is an approximation of the total derivative in the reduced eigenvector space:

$\mathrm{d} t=\sum_{j=1}^{n \operatorname{comp}} \frac{\delta t}{\delta \boldsymbol{v}_{j}} d \boldsymbol{v}_{j}$ 
with

$$
\begin{aligned}
\frac{\delta t}{\delta \boldsymbol{v}_{j}} & \approx \frac{t(0,0, \ldots, 1, \ldots, 0)-t(0,0, \ldots, 0)}{1} \\
& =t_{\text {mean }+\boldsymbol{v}_{j}}-t_{\text {mean }}=\Delta t_{\boldsymbol{v}_{j}}
\end{aligned}
$$

and

$d \boldsymbol{v}_{j}=\mathrm{d} \boldsymbol{T} \cdot \boldsymbol{v}_{j}=\left(\boldsymbol{T}-\boldsymbol{T}_{\text {mean }}\right)^{\top} \cdot \boldsymbol{v}_{j}=c_{j}$.

In order to account for non-linear effects, we can extend the approximation of $t$ by its second derivative with respect to $\boldsymbol{v}_{j}$ :

$\mathrm{d}^{2} t=\sum_{j=1}^{n \text { comp }} \frac{\delta^{2} t}{\delta v_{j}^{2}} d v_{j}^{2}$

with

$\frac{\delta^{2} t}{\delta \boldsymbol{v}_{j}^{2}}=t_{\text {mean }+\boldsymbol{v}_{j}}+t_{\text {mean }-\boldsymbol{v}_{j}}-2 t_{\text {mean }}=\Delta^{2} t_{\boldsymbol{v}_{j}}$

and

$d v_{j}^{2}=c_{j}^{2}$

Acknowledgements. This work was carried out within the framework of the ESA ESTEC-funded project "Atmospheric corrections for fluorescence signal and surface pressure retrieval over land" (contract nr. 4000102733).

Edited by: J. Joiner

\section{References}

Barton, I. J. and Scott, J. C.: Remote measurement of surface pressure using absorption in the oxygen A-band, Appl. Opt., 25, 3502-3507, 1986.

Breon, F. M. and Bouffies, S.: Land surface pressure estimate from measurements in the oxygen A absorption band, J. Appl. Meteor., 35, 69-77, 1996.

Delwart, S., Preusker, R., Bourg, L., Santer, R., Ramon, D., and Fischer, J.: MERIS inflight spectral calibration, Int. J. Remote Sens., 28, 479-496, 2007.

Fell, F. and Fischer, J.: Numerical simulation of the light field in the atmosphere-ocean system using the matrix-operator method, J. Quant. Spectrosc. Radiat. Transfer, 3, 351-388, 2001.

Gao, B.-C. and Kaufman, Y. J.: Water vapor retrievals using Moderate Resolution Imaging Spectroradiometer (MODIS) near-infrared channels, J. Geophys. Res., 108, 4389, doi:10.1029/2002JD003023, 2003.
Hollstein, A. and Fischer, J.: Radiative transfer solutions for coupled atmosphere ocean systems using the matrix operator technique, J Quant. Spectros. Radiat. Transfer, 113, 536-548, doi:10.1016/j.jqsrt.2012.01.010, 2012.

Lindstrot, R., Preusker, R., and Fischer, J.: The retrieval of land surface pressure from MERIS measurements in the oxygen A band., J. Atmos. Oceanic Technol., 26, 1367-1377, 2009.

Lindstrot, R., Preusker, R., and Fischer, J.: The empirical correction of stray light in the MERIS oxygen A band channel, J. Atmos. Oceanic Technol., 27, 1185-1194, 2010.

Lindstrot, R., Preusker, R., Diedrich, H., Doppler, L., Bennartz, R., and Fischer, J.: 1D-Var retrieval of daytime total columnar water vapour from MERIS measurements, Atmos. Meas. Tech., 5, 631646, doi:10.5194/amt-5-631-2012, 2012.

Liou, K.: An Introduction to Atmospheric Radiation, International Geophysics Series, Academic Press, 2002.

Liu, X., Smith, W. L., Zhou, D. K., and Larar, A.: Principal component-based radiative transfer model for hyperspectral sensors: theoretical concept, Appl. Opt., 45, 201-209, 2006.

Matricardi, M.: A principal component based version of the RTTOV fast radiative transfer model, Q. J. Roy. Meteorol. Soc., 136, 1823-1835, doi:10.1002/qj.680, 2010.

Mitchell, R. M. and O'Brien, D. M.: Error Estimates for Passive Satellite Measurement of Surface Pressure Using Absorption in the A Band of Oxygen, J. Atmos. Sci., 44, 1981-1990, 1987.

Natraj, V., Jiang, X., Shia, R., Huang, X., Margolis, J. S., and Yung, Y. L.: Application of principal component analysis to high spectral resolution radiative transfer: A case study of the $\mathrm{O}_{2}$ A band, J. Quant. Spectros. Radiat. Transfer, 95, 539-556, doi:10.1016/j.jqsrt.2004.12.024, 2005.

Natraj, V., Shia, R.-L., and Yung, Y. L.: On the use of principal component analysis to speed up radiative transfer calculations, J. Quant. Spectros. Radiat. Transfer, 111, 810-816, doi:10.1016/j.jqsrt.2009.11.004, 2010.

O'Brien, D., Mitchell, R., English, S., and Da Costa, G.: Airborne Measurements of Air Mass from $\mathrm{O}_{2}$ A-Band Absorption Spectra, J. Atmos. Oceanic Technol., 15, 1272-1286, 1998.

Peixoto, J. and Oort, A.: Physics of Climate, American Institute of Physics, 1992.

Rothman, L., Gordon, I., Barbe, A., Benner, D., Bernath, P., Birk, M., Boudon, V., Brown, L., Campargue, A., Champion, J.-P., Chance, K., Coudert, L., Dana, V., Devi, V., Fally, S., Flaud, J.M., Gamache, R., Goldman, A., Jacquemart, D., Kleiner, I., Lacome, N., Lafferty, W., Mandin, J.-Y., Massie, S., Mikhailenko, S., Miller, C., Moazzen-Ahmadi, N., Naumenko, O., Nikitin, A., Orphal, J., Perevalov, V., Perrin, A., Predoi-Cross, A., Rinsland, C., Rotger, M., Simeckova, M., Smith, M., Sung, K., Tashkun, S., Tennyson, J., Toth, R., Vandaele, A., and Auwera, J. V.: The HITRAN 2008 molecular spectroscopic database, J. Quant. Spectrosc. Radiat. Transfer, 110, 533-572, doi:10.1016/j.jqsrt.2009.02.013, 2009.

Schneising, O., Buchwitz, M., Burrows, J. P., Bovensmann, H., Bergamaschi, P., and Peters, W.: Three years of greenhouse gas column-averaged dry air mole fractions retrieved from satellite - Part 2: Methane, Atmos. Chem. Phys., 9, 443-465, doi:10.5194/acp-9-443-2009, 2009. 